\title{
On the comparative biology of mammalian telomeres: Telomere length co-evolves with body mass, lifespan and cancer risk
}

\author{
Michael Pepke ${ }^{1}$ and Dan Eisenberg ${ }^{2}$ \\ ${ }^{1}$ Norges teknisk-naturvitenskapelige universitet \\ ${ }^{2}$ University of Washington
}

August 7, 2020

\begin{abstract}
Telomeres, the short repetitive DNA sequences that cap the ends of linear chromosomes, shorten during cell division and are implicated in senescence in most species. Telomerase can rebuild telomeres but is repressed in many mammals that exhibit replicative senescence, presumably as a tumor suppression mechanism. It is therefore important that we have an accurate understanding of the co-evolution of telomere biology and life-history traits that has shaped the diversity of senescence patterns across species. Gomes et al. (2011) produced a large data set on telomere length (TL), telomerase activity, body mass and lifespan among 57 mammal species. We re-analyzed their data using the same phylogenetic multiple regressions and with several additional analyses to test the robustness of findings. We found substantial inconsistencies in our results compared to Gomes et al.'s. Consistent with Gomes et al. we found an inverse association between TL and lifespan. Contrary to the analyses in Gomes et al., we found a generally robust inverse association between TL and mass, and only weak non-robust evidence for an association between telomerase activity and mass. These results suggest that shorter TL may have been selected for in larger and longer-lived species-likely as a mechanism to suppress cancer. We support this hypothesis by showing that longer telomeres predict higher cancer risk across 22 species. Furthermore, we find that domesticated species have longer telomeres. Our results call into question past interpretations of the co-evolution of telomere biology and life-history traits and stress the need for careful attention to model construction.
\end{abstract}

\section{INTRODUCTION}

Telomeres are short repetitive DNA sequences that cap the ends of linear chromosomes and are highly conserved across most eukaryotic organisms (Meyne et al., 1989; Blackburn, 1991). Telomeres shorten during cell replication and may be further degraded due to oxidative stress (von Zglinicki, 2002). When telomeres get too short, the cell typically ceases to replicate. The enzyme telomerase can rebuild telomeres (Greider, 1990) but is normally repressed in somatic cells of many mammals, presumably as a tumor suppression mechanism (Gomes et al., 2010; Tian et al., 2018). In the absence of telomerase, telomere shortening triggers cell senescence, which can prevent proliferation of precancerous cells (Campisi, 2001). Thus, replicative senescence has been interpreted as an adaptive tumor-suppression mechanism (e.g. Campisi, 2001).

Cross-species analyses have furthered understandings of evolutionary trade-offs shaping telomere length (TL) and telomerase activity. Haussmann et al. (2003) showed that telomere loss rate, but not TL, was inversely correlated with maximum lifespan in birds, which has since been corroborated across tetrapod species (Dantzer \& Fletcher, 2015; Sudyka et al., 2016; Tricola et al., 2018, Whittemore et al., 2019; Pepke \& Eisenberg, 2020). Seluanov et al. (2007) found that telomerase activity was negatively correlated with body mass, but not lifespan in rodents. Since, all else equal, increased telomerase activity is expected to lead to slower telomere erosion, Seluanov et al.'s results apparently contrast with the results on telomere shortening rates reported above. Seluanov et al. also found no significant associations of TL with body mass or lifespan. In an analysis of 57 mammalian species, Gomes et al. (2011) reported a negative relationship 
between telomerase activity and mass, but not lifespan across mammals. Furthermore, Gomes et al. found that lifespan was inversely correlated with TL while accounting for body mass, but there was no independent association between TL and mass. Here we build on these past findings, first trying to replicate the analyses of Gomes et al., then proceeding to further examine how telomere biology may influence cross-species variation in cancer risk and finally how telomere biology may have been shaped by selection related to domestication.

Telomere biology is widely assumed to influence cancer risk and thus for different species to evolve different telomere dynamics in response to differing fitness costs of cancer. Cancer occurs in almost all vertebrates, but among wild animals, cancer incidence rates are little studied, but seem to vary considerably (Effron et al. 1977; Pesavento et al. 2018; Albuquerque et al. 2018; Boddy et al. 2020). For instance, cancer is rarely reported in long-lived whales, bats or naked mole-rats, while rats and mice are known to be much more prone to cancer (Albuquerque et al. 2018). Different species have evolved different anti-cancer strategies and mechanisms; e.g. long-lived species that reproduce late in life are expected to be under stronger selection pressure to evolve efficient cancer defenses compared to short-lived species (Weinstein and Ciszek, 2002; Seluanov et al. 2008; 2018). Despite larger and longer-lived species having more cells that exist for a longer duration, they do not show higher cancer rates than smaller, short-lived species (Peto et al. 1975). This phenomenon, known as 'Peto's Paradox', is generally assumed to be explained by species with larger body sizes and longer lives having increased fitness costs from cancer development. Correspondingly larger and longer-lived species are thought to tend to evolve shorter telomeres and decreased telomerase activity (Caulin \& Maley, 2011; Gomes et al., 2011; Tian et al., 2018; Risques \& Promislow, 2018). Thus, longer telomeres are predicted to increase the risk of cancer (e.g. Aviv et al., 2017). However, there are reasons to question the associations between telomere length and cancer risk (reviewed in Eisenberg \& Kuzawa, 2018). Shorter telomeres can cause chromosomal instability that promotes cancer. Longer telomeres improve immune function, which is important in combatting many infectious diseases which cause cancers and in combatting the development of malignancies. To better characterize the association between telomere biology and cancer, using cross-species datasets we examine whether longer telomeres and telomerase activity predict increased cancer incidence as is often assumed.

In both mice (Mus musculus and Peromyscus leucopus) and pearl millet (Pennisetum glaucum), domesticated strains have been observed to have longer TLs than their wild counterparts (Bickle et al., 1998; Hemann \& Greider, 2000; Manning et al., 2002; Weinstein and Ciszek, 2002; Sridevi et al., 2002; Kotrschal et al., 2007; Seluanov et al., 2008). Multiple explanations for this putative association between domestication and longer TL have been suggested (Manning et al., 2002; Weinstein and Ciszek, 2002; Eisenberg, 2011). To better characterize how general the association between domestication and TL is, we examine this association across a cross-species dataset including 9 domesticated and 48 non-domesticated mammalian species.

Our analyses primarily build on a cross-species dataset generated by Gomes et al. (2011). Gomes et al. measured mean telomere length (TL) and telomerase activity among 43 mammal species and combined with comparable published measurements on 14 species. Gomes et al. then examined relationships between TL, telomerase activity, body mass and maximum lifespan. As a first step to test our hypotheses regarding domestication and cancer risk, we aimed to replicate the findings from Gomes et al.'s analysis. We found considerably discrepant results. To establish the robustness of the findings we conducted additional sensitivity analyses. Thus, in this manuscript we first present our re-analysis of Gomes et al. and try to explain these discrepant results. Then we proceed to test whether TL and TA predict cancer risk and that TL was elongated by domestication.

\section{MATERIALS AND METHODS}

\section{Replication of analyses in Gomes et al. (2011)}

Gomes et al. (2011) measured mean TL in kb in adult individuals using the telomere restriction fragment (TRF) method. Telomerase activity was measured in cultured fibroblasts relative to an adenocarcinoma cell line (\%H1299). Following Gomes et al. (2011) we used the best species-level mammal supertree from Bininda-Emonds et al. (2007), which is available within the R package 'PhyloOrchard' (O'Meara et al., 
2012). The phylogeny was pruned to match the dataset using the R package 'ape' (Paradis \& Schliep, 2018). We used the phylogenetic generalized least squares method (Grafen, 1989) implemented as the 'pgls' function in the package 'caper' (Orme et al., 2018) to perform the phylogenetic regressions. Pagel's $\lambda$ was estimated with default bounds (0-1) using maximum likelihood. We used estimates of adult body mass $(\mathrm{kg})$ and maximum lifespan (years) reported in Gomes et al. (2011), which were derived from the AnAge: The Animal Ageing and Longevity Database (Tacutu et al. 2018). Because some of these were different from current estimates in AnAge we compiled new values for a separate re-analysis (see Supporting Information Table S2). Following Gomes et al. (2011) TL, mass and lifespan were all $\log _{10}$-transformed. We performed two multivariate phylogenetic regressions (outlined in Fig. 2 in Gomes et al., 2011) of body mass (response variable) predicted by telomerase activity and TL, and of maximum lifespan (response variable) predicted by telomerase activity, TL and body mass. The phylogeny and TL evolution were visualized using the 'phytools' package (Revell, 2012) in which ancestral states were estimated following Felsenstein (1985) but using maximum likelihood with the function 'fastAnc'.

\section{Associations between neoplasia incidence and telomere biology}

We compiled neoplasia incidence rates in 7 species of mammals from a medically curated postmortem database published by Boddy et al. (2020) in addition to 15 species from various sources in the literature reviewed by Albuquerque et al. (2018) and shown in Table S10. In all studies, the number of individuals with abnormal growth including both benign and malignant tumors (neoplasia) out of the total number of necropsies were used to define the neoplasia prevalence rate (\%). For the large treeshrew (Tupaia tana ) neoplasia rate is unknown, so we used the estimate reported for the closely related common treeshrew (Tupaia glis ). Due to small samples sizes, neoplasia data were combined in Boddy et al. (2020) for the Asian and African elephant (Elephas maximus and Loxodonta africana), so we used the average TL, mass and lifespan of the two species, which are similar (Table S9), in subsequent analyses. Boddy et al. (2020) also provided estimates of lifetime prevalence of malignant neoplasms (invading surrounding normal tissue) of 29 mammal species, allowing us to test if neoplasia prevalence is generally a good predictor of malignancy prevalence (i.e. cancer rate in \%). Most neoplasia data are from wild animals kept in captivity with no age or sex information, and not all tissues were investigated during necropsies, thus the data only provide crude estimates of the natural rate of spontaneous cancers.

Species mean telomere length measured using the TRF method allows comparisons of absolute TLs and were collected from both Gomes et al. (2011) and Seluanov et al. (2007) to increase sample size (Table S10). Six overlapping species in these two studies show that the TL measurements are highly correlated $\left(\mathrm{R}^{2}=0.9431\right)$, but consistently higher in Seluanov et al. (2007). We therefore used an ordinary linear regression of TLs of the overlapping species to adjust measurements of the four species (Table S10) from Seluanov et al. (2007) to match Gomes et al. (2011) using the formula: $0.6617 \mathrm{x}$ TL - $2.4454 \mathrm{~kb}$. Telomerase activity was measured using different methodologies, so we can only report whether telomerase was present (1) or absent (0) in the somatic cells of the species. We then test phylogenetic bivariate associations between neoplasia rate and TL, telomerase expression, adult body mass and maximum lifespan (obtained from the AnAge database, Tacutu et al., 2018), and whether TL and/or telomerase expression predict neoplasia rates (response variable) using phylogenetic multiple regression as described above.

\section{Effect of domestication on telomere length}

We assigned a binary variable (0/1) indicating the nine species in Gomes et al. (2011) that have a long history of human domestication and captive-breeding (Scherf, 2000; Wilkins et al., 2014), see Table S9: cow (Bos taurus), sheep (Ovis aries), pig (Sus scrofa), dromedary camel (Camelus dromedarius), horse (Equus caballus ), dog (Canis lupus familiaris ), European white rabbit (Oryctolagus cuniculus ), laboratory mouse (Mus musculus ), and laboratory rat (Rattus norvegicus ). We first tested whether TL (response variable) is different in domesticated species using a phylogenetic bivariate regression. Since TL is also hypothesized to be influenced by telomerase activity, body mass and lifespan we also tested the effect of domestication on TL (response variable) while accounting for telomerase activity, body mass and lifespan (predictor variables)

using two phylogenetic multiple regressions, as described above. All analyses were performed in R (R Core 
Team, 2020).

\section{RESULTS}

Replication of analyses in Gomes et al. (2011)

Presented in Table 1 are the results reported by Gomes et al. (2011) and our re-analysis - both using two phylogenetic multiple regression models. Consistent with Gomes et al. (2011), we found that both mass and TL significantly predicted lifespan. However, the remaining results are all substantially discordant. While Gomes et al. (2011) found no association between telomerase activity and lifespan, we observed a significantly positive association. In predicting body mass, Gomes et al. (2011) found that telomerase activity was a significant predictor, but TL was not. We found the opposite: telomerase activity did not predict mass, but longer TL predicted decreased body mass. Using current estimates for mass and lifespan from the AnAge database (Table S9) did not qualitatively alter the findings described above except the association between telomerase activity and lifespan was attenuated $(\beta=0.0036, \mathrm{p}=0.0594$, Table S2).

Gomes et al. (2011) reported a series of bivariate plots (their Fig. 4) for key variables in their analyses along with p-values. These p-values are identical to those presented in their Fig. 2 (our Table 1), strongly suggesting that these significance values were from phylogenetic multiple regressions, not phylogenetic bivariate regressions. A casual reader is likely to interpret these p-values as bivariate associations. Here, we present phylogenetic linear bivariate regression analyses of TL, body mass, maximum lifespan, and telomerase activity to examine the sensitivity of the multiple regression results and more clearly define these bivariate associations.

The bivariate results (Fig. 1) are qualitatively consistent with our multiple regression results, except lifespan did not show any association with telomerase activity (Fig. 1d). Based on these bivariate results, a $1 \%$ increase in body mass predicts a $0.08 \%$ decrease in TL (Fig. 1a), while a $1 \%$ increase in lifespan predicts a $0.43 \%$ reduction in TL (Fig. 1b). The co-evolution of TL and body mass is illustrated in the phylogeny in Fig. 2.

In an effort to further reconstruct the reasons for our discrepant results with those of Gomes et al. and explore the sensitivity of our analyses to modeling strategies, we experimented with alternative transformations of the telomerase activity measurements (see Supporting Information). Briefly, we find that in multiple regression models, as telomerase activity is first log-transformed (Table S3) and then binary transformed (Table S4), TL becomes less and less predictive of either mass or lifespan and the correlation between TL and telomerase activity increases (Table S5). When the bivariate association of mass predicting binary telomerase activity $(0 / 1)$ is modelled in a phylogenetic logistic regression (see Supporting Information), complete telomerase repression is significantly associated with higher body mass with an inflection point at $0.8 \mathrm{~kg}$ (Fig. S1).

\section{Associations between neoplasia incidence and telomere biology}

We found that the prevalence of neoplasia is a good predictor of malignancy prevalence $\left(\mathrm{R}^{2}=0.90\right.$, $\beta=0.7735 \pm 0.0489, p<0.001$, Fig. S2) across 29 mammal species included in Boddy et al. (2020). Because neoplasia prevalence was available for more species, analyses proceeded using this variable.

As expected, neoplasia rate was positively associated with TL (Fig. 3a) and binary telomerase activity (Fig. $3 \mathrm{~d}$, marginally significant trend) across the 22 mammal species shown in Table S9. Thus, a $1 \%$ increase in TL predicts an absolute increase in neoplasia incidence of $0.21 \%$ (Fig. 3a). Neoplasia rate was negatively associated with lifespan (Fig 3b). A $1 \%$ increase in maximum lifespan predicts an absolute decrease in neoplasia incidence of $0.14 \%$ (Fig. 3b). Species with some telomerase activity (1) are predicted to show an absolute increase in neoplasia incidence of $20.6 \%$ (Fig. 3d). There was no association between neoplasia rate and mass (Fig. 3c). There was a marginal significant positive association between TL and neoplasia rate, when accounting for binary telomerase activity (Table 2).

Neoplasia rates could be biased towards higher estimates in domesticated species due to the easier observation of large numbers of individuals (Nagy et al., 2007; Ewald \& Ewald, 2015; Boddy et al., 2020), which 
could be associated with increased lifespan and senescence in captivity (Tidière et al., 2016). We therefore tested, in a post hoc analysis, whether the effect of TL on neoplasia rate was robust to controlling for potential domestication effects on neoplasia rates (Table S6) or if the effect of TL varied with domestication status (Table S7). TL remained significantly positively associated with neoplasia rate when controlling for domestication status and there was no effect of domestication status on neoplasia rate (Table S6). The effect of TL on neoplasia rate did not vary with domestication status (Table S7).

\section{Effect of domestication on telomere length}

The arithmetic mean TL was $24.8 \mathrm{~kb}$ for domesticated species and $20.3 \mathrm{~kb}$ for non-domesticated species in the Gomes et al. (2011) dataset. Controlling for phylogenetic non-independence, there was a marginally significant positive trend, towards longer telomeres in domesticated species $\left(\beta_{\text {domestication }}=0.1467 \pm 0.0774\right.$, $\mathrm{p}=0.0633)$. Telomeres were significantly longer in domesticated species $\left(\beta_{\text {domestication }}=0.1416 \pm 0.0615\right.$, $\mathrm{p}=0.0254$ ) when controlling for telomerase activity, body mass and lifespan (Table 3). Thus, domestication is predicted to increase TL by $38.5 \%$ compared to non-domesticated species.

Excluding the nine domesticated species from the analyses presented in Table 1 above did not qualitatively alter our findings (see Table S8), but the positive association between telomerase activity and lifespan was attenuated $\left(\beta_{\text {telomerase activity }}=0.0034 \pm 0.0018, \mathrm{p}=0.0703\right)$.

\section{DISCUSSION}

Our re-analysis of the data from Gomes et al 2011 demonstrates that telomere length has evolved to be shorter with increasing body mass and lifespan, across 57 species of mammals spanning 15 orders (Fig. 2). This supports the hypothesis that short telomeres evolved in large, long-lived mammals to enable replicative senescence as a mechanism to ameliorate cancer risk conferred by a larger number of cells and longer time to accumulate oncogenic mutations (Gorbunova et al., 2014; Risques \& Promislow, 2018). Alternatively, since TL was measured in adults, larger species may erode their telomeres faster than small species, giving rise to the negative correlation between TL and body mass (Monaghan \& Ozanne, 2018). However, larger species have been shown to have lower telomere shortening rates across 9 tetrapod species, but this association was driven by inclusion of a domesticated mouse strain (Pepke \& Eisenberg, 2020). Since telomere loss rates tend to be slower in longer lived species (Haussmann et al., 2003; Dantzer \& Fletcher, 2015), TLs at conception are likely to be even longer in shorter-lived species than estimated in adulthood.

In contrast to our findings, past studies have shown no significant associations between TL with mass or lifespan across 15 rodent species (Seluanov et al., 2007) or between TL and lifespan of 19 bird species (Tricola et al., 2018). These differences might be explained by these rodents and birds being relatively small and short-lived compared to many of the species included here. Among 8 species of relatively large mammals and birds (all $>0.5 \mathrm{~kg}$ ), longer early-life TL predicted longer lifespan (the opposite of our findings), but only when accounting for body mass and telomere shortening rate (Pepke \& Eisenberg, 2020). Gomes et al. (2011) failed to show the association between TL and mass that we found analyzing the same dataset. While the reasons for our differing results with Gomes et al. are unclear, they may be driven by a transformation of telomerase activity in the multivariate model (discussed below). Regardless, broader taxonomic studies that are attentive to model construction are needed to unravel the generality of these patterns.

Our re-analysis of Gomes et al. (2011) showed that telomerase activity had only inconsistent associations with lifespan and body mass (Tables 1 and S1-4), which call into question past results suggesting that high telomerase activity has been selected against in larger and longer-lived species. We found some hints that telomerase activity was inversely associated with mass when telomerase was either log-transformed or binary transformed (Tables S3-4 and Fig. S1). Thus, the associations between telomere biology, mass and lifespan are sensitive to modeling strategies. While we found an inverse association of binary telomerase activity with mass, the biological meaning of binary coded telomerase activity is unclear. Perhaps having any telomerase activity increases the level of cancer risk, but the particular level of telomerase activity greater than zero does not change cancer risk. This might suggest that telomerase activity variation greater than zero has no meaningful effects on the phenotype under selection with changing body mass. In contrast, Seluanov 
et al. (2007) found a strong negative linear correlation between (untransformed) telomerase activity and body mass across rodent species. Seluanov et al.'s analysis differed from this study in that they measured telomerase activity in spleen, liver, and kidney tissues, while this study depends on telomerase from cultured cells. Additionally, the rodents in Seluanov et al.'s analysis all expressed telomerase and had long telomeres (most species $>20 \mathrm{~kb}$ ), while this analysis includes many species without detectable telomerase activity and with shorter TLs. The fact that Seluanov et al.'s analysis showed non-transformed telomerase activity had an association with mass among animals with only non-zero telomerase activity argues against using telomerase activity as a binary variable. Furthermore, small levels of telomerase activity in normal stem cells is thought to be insufficient to fully maintain telomere lengths (Shay \& Wright, 2010).

Telomere length was a strong predictor of neoplasia rates across 22 species spanning 11 orders (Fig. 3a). Neoplasia rates are a good proxy for malignancy rates (Fig. S2). In turn, malignancy rates likely reflect the incidence of development of cancers with fitness consequences (Ewald \& Ewald, 2015). Thus, natural selection against longer telomeres may be driven by cancer-related mortality. Our findings are consistent with the hypothesis that shorter telomeres have evolved as a cancer resistance mechanism across mammals. Larger and longer-lived species did not have higher neoplasia incidence rates (Fig. 3c), which is a demonstration of the well-known Peto's paradox. The negative correlation that we found between TL and body mass may partly underlie Peto's paradox; i.e. larger species have evolved shorter telomeres as an anti-cancer mechanism to compensate for otherwise higher cancer risks (Caulin \& Maley 2011). The optimal TL in mammals may therefore have been shaped by a trade-off between the increased cancer risk of long telomeres and the increased susceptibility to age-dependent degenerative diseases conferred by short telomeres (Tian et al., 2018; Aviv et al., 2017). We also found some evidence that binary telomerase activity was associated with higher cancer rates (Fig. 3d), although this effect was not significant when controlling for TL (Table 2). High telomerase activity is a prerequisite for most cancer cells (Wright \& Shay, 2005) and may decrease the threshold for cell immortalization leading to increased cancer risk in species with somatic telomerase activity.

Although species with short telomeres have lower cancer incidence rates, some species with long telomeres appear to have lower cancer rates than expected given their TL. This may be explained by the evolution of various telomere-independent cancer defense mechanisms in these typically long-lived species (Seluanov et al., 2018; Erten \& Kokko, 2020). The most striking outlier in our analysis is the tiger (Panthera tigris ), having a large body size $(162 \mathrm{~kg})$ despite having very long telomeres $(50 \mathrm{~kb})$, high telomerase activity (10\%), but a moderate neoplasia incidence rate (18\%), suggesting the potential for discovery of novel tumor suppressor mechanisms within this species.

We provided the first evidence that telomeres are longer across multiple domesticated species spanning 5 orders (mainly members of Artiodactyla, but also of Perissodactyla, Carnivora, Rodentia and Lagomorpha). Long telomeres may form part of a "domestication syndrome" in mammals (Darwin, 1868; Wilkins et al., 2014), although the mechanisms generating this trait change are not well-known (Eisenberg, 2011). Alternatively, long telomeres were ancestral in domesticated species, but somehow connected to the early stages of domestication (Lord et al., 2020). We found that domestication has resulted in an estimated $38.5 \%$ increase in TL (Table 3), which is equivalent to the effect an $98.4 \%$ decrease in body mass would have on TL (Fig. 1a). There does not appear to be a single directional change in body sizes of domesticated animals (Lord et al., 2020). However, domesticated species may have experienced changes related to fertility, reproductive lifespan, growth rate, degree of inbreeding, parasite load and energetic constraints (Diamond, 1997; Lord et al., 2020), which are all effects known to shape intraspecific variation in TL (Weinstein and Ciszek, 2002; Manning et al., 2002; Bebbington et al., 2016; Monaghan \& Ozanne, 2018; Sudyka, 2019; Giraudeau et al., 2019). Comparisons of TL and other phenotypes in domesticated species with their wild or feral cousins might provide more insights into the particular causes of TL changes with domestication.

Our re-analyses of the valuable dataset generated by Gomes et al. (2011) showed considerably divergent results from those shown by Gomes et al. (2011) and that many results are not robust to varying modeling strategies. Unlike Gomes et al. (2011), we showed that telomere length decreases with increasing body mass. While Gomes et al.'s results suggest a reduction in telomerase activity with increasing body mass, we only 
found weak evidence for this. Our results suggest that the co-evolution of telomere biology with lifespan and body mass across species needs to be reconsidered. The negative association between TL and mass and lifespan could be explained by the increased cancer risk we found in species with longer telomeres. Finally, we found that domesticated species appear to have evolved longer telomeres - suggesting new avenues to understand telomere evolution through closer examination of the domestication process and artificial selection experiments.

Acknowledgements: We thank Amy Klegarth for collaboration on earlier exploration of these data and Jerry Shay and Chris Venditti for collegial discussion of our discrepant results. We thank Pat Monaghan and Dan Nussey for organizing the Diversity in Telomere Dynamics Workshop, which facilitated the authors meeting and collaborating on this project. M.L.P. thanks the Research Council of Norway for funding through its Centres of Excellence scheme (223257).

\section{REFERENCES}

Albuquerque, T. A. F., Drummond do Val, L., Doherty, A., \& de Magalhães, J. P. (2018). From humans to hydra: patterns of cancer across the tree of life. Biological Reviews, 93 (3), 1715-1734. doi:10.1111/brv.12415

Aviv, A., Anderson, J. J., \& Shay, J. W. (2017). Mutations, cancer and the telomere length paradox. Trends in Cancer, 3 (4), 253-258. doi:https://doi.org/10.1016/j.trecan.2017.02.005

Bebbington, K., Spurgin, L. G., Fairfield, E. A., Dugdale, H. L., Komdeur, J., Burke, T., \& Richardson, D. S. (2016). Telomere length reveals cumulative individual and transgenerational inbreeding effects in a passerine bird. Mol Ecol, 25 (12), 2949-2960. doi:10.1111/mec.13670

Bickle, C. A., Cantrell, M., Austad, S., \& Wichman, H. (1998).Effects of domestication on telomere length in Mus musculus and Peromyscus maniculatus. Paper presented at the Faseb Journal.

Bininda-Emonds, O. R. P., Cardillo, M., Jones, K. E., MacPhee, R. D. E., Beck, R. M. D., Grenyer, R., . . Purvis, A. (2007). The delayed rise of present-day mammals. Nature, 446 (7135), 507-512. doi:10.1038/nature05634

Blackburn, E. H. (1991). Structure and function of telomeres.Nature, 350 (6319), 569-573. doi:10.1038/350569a0

Boddy, A. M., Abegglen, L. M., Pessier, A. P., Schiffman, J. D., Maley, C. C., \& Witte, C. (2020). Lifetime cancer prevalence and life history traits in mammals. Evolution, Medicine, and Public Health . doi:10.1093/emph/eoaa015

Campisi, J. (2001). Cellular senescence as a tumor-suppressor mechanism. Trends in Cell Biology, 11 (11), S27-S31. doi:https://doi.org/10.1016/S0962-8924(01)02151-1

Caulin, A. F., \& Maley, C. C. (2011). Peto's Paradox: evolution's prescription for cancer prevention. Trends in Ecology $\&$ Evolution, 26 (4), 175-182. doi:https://doi.org/10.1016/j.tree.2011.01.002

Dantzer, B., \& Fletcher, Q. E. (2015). Telomeres shorten more slowly in slow-aging wild animals than in fast-aging ones. Experimental gerontology, 71 , 38-47. doi:https://doi.org/10.1016/j.exger.2015.08.012

Darwin, C. (1868). The variation of animals and plants under domestication (Vol. 2). Cambridge: Cambridge University Press.

Diamond, J. M. (1997). Guns, germs, and steel . New York: Norton.

Effron, M., Griner, L., \& Benirschke, K. (1977). Nature and rate of neoplasia found in captive wild mammals, birds, and reptiles at necropsy. JNCI: Journal of the National Cancer Institute, 59 (1), 185-198. doi:10.1093/jnci/59.1.185

Eisenberg, D. T. A. (2011). An evolutionary review of human telomere biology: The thrifty telomere hypothesis and notes on potential adaptive paternal effects. American Journal of Human Biology, 23 (2), 149-167. 
doi:10.1002/ajhb.21127

Eisenberg, D. T. A., \& Kuzawa, C. W. (2018). The paternal age at conception effect on offspring telomere length: mechanistic, comparative and adaptive perspectives. Philosophical Transactions of the Royal Society B: Biological Sciences, 373 (1741), 20160442. doi:10.1098/rstb.2016.0442

Ewald, P. W., \& Swain Ewald, H. A. (2015). Infection and cancer in multicellular organisms. Philosophical Transactions of the Royal Society B: Biological Sciences, 370 (1673), 20140224. doi:10.1098/rstb.2014.0224

Felsenstein, J. (1985). Phylogenies and the comparative method. The American Naturalist, 125 (1), 1-15. doi:Doi 10.1086/284325

Giraudeau, M., Heidinger, B., Bonneaud, C., \& Sepp, T. (2019). Telomere shortening as a mechanism of long-term cost of infectious diseases in natural animal populations. Biology Letters, 15 (5), 20190190. doi:10.1098/rsbl.2019.0190

Gomes, N. M., Ryder, O. A., Houck, M. L., Charter, S. J., Walker, W., Forsyth, N. R., . . Wright, W. E. (2011). Comparative biology of mammalian telomeres: hypotheses on ancestral states and the roles of telomeres in longevity determination. Aging Cell, 10 (5), 761-768. doi:10.1111/j.1474-9726.2011.00718.x

Gomes, N. M. V., Shay, J. W., \& Wright, W. E. (2010). Telomere biology in Metazoa. Febs Letters, 584 (17), 3741-3751. doi:10.1016/j.febslet.2010.07.031

Gorbunova, V., Seluanov, A., Zhang, Z., Gladyshev, V. N., \& Vijg, J. (2014). Comparative genetics of longevity and cancer: insights from long-lived rodents. Nature Reviews Genetics, 15 (8), 531-540. doi:10.1038/nrg3728

Greider, C. W. (1990). Telomeres, telomerase and senescence.Bioessays, 12 (8), 363-369. doi:10.1002/bies.950120803

Haussmann, M. F., Winkler, D. W., O’Reilly, K. M., Huntington, C. E., Nisbet, I. C. T., \& Vleck, C. M. (2003). Telomeres shorten more slowly in long-lived birds and mammals than in short-lived ones.Proceedings of the Royal Society of London. Series B: Biological Sciences, 270 (1522), 1387-1392. doi:10.1098/rspb.2003.2385

Hemann, M. T., \& Greider, C. W. (2000). Wild-derived inbred mouse strains have short telomeres. Nucleic Acids Research, 28 (22), 4474-4478. doi:10.1093/nar/28.22.4474

Klegarth, A. R., \& Eisenberg, D. T. A. (2018). Mammalian chromosome-telomere length dynamics. Royal Society Open Science, 5 (7), 180492. doi:10.1098/rsos.180492

Kotrschal, A., Ilmonen, P., \& Penn, D. J. (2007). Stress impacts telomere dynamics. Biology Letters, 3 (2), 128-130. doi:10.1098/rsbl.2006.0594

Larson, G., \& Fuller, D. Q. (2014). The evolution of animal domestication. Annual Review of Ecology, Evolution, and Systematics, 45 (1), 115-136. doi:10.1146/annurev-ecolsys-110512-135813

Lord, K. A., Larson, G., Coppinger, R. P., \& Karlsson, E. K. (2020). The history of farm foxes undermines the animal domestication syndrome.Trends in Ecology $\&$ Evolution, 35 (2), 125-136. doi:https://doi.org/10.1016/j.tree.2019.10.011

Manning, E. L., Crossland, J., Dewey, M. J., \& Van Zant, G. (2002). Influences of inbreeding and genetics on telomere length in mice.Mammalian Genome, 13 (5), 234-238. doi:10.1007/s003350020027

Meyne, J., Ratliff, R. L., \& Moyzis, R. K. (1989). Conservation of the human telomere sequence (TTAGGG)n among vertebrates. Proceedings of the National Academy of Sciences of the United States of America, 86 (18), 7049-7053. doi:10.1073/pnas.86.18.7049 
Monaghan, P., \& Ozanne, S. E. (2018). Somatic growth and telomere dynamics in vertebrates: relationships, mechanisms and consequences.Philosophical Transactions of the Royal Society B: Biological Sciences, 373 (1741), 20160446. doi:10.1098/rstb.2016.0446

Nagy, J. D., Victor, E. M., \& Cropper, J. H. (2007). Why don't all whales have cancer? A novel hypothesis resolving Peto's paradox.Integrative and Comparative Biology, 47 (2), 317-328. doi:10.1093/icb/icm062

Pepke, M. L., \& Eisenberg, D. T. A. (2020). Accounting for phylogenetic relatedness in cross-species analyses of telomere shortening rates.Experimental Results, 1, e11. doi:10.1017/exp.2020.18

Pesavento, P. A., Agnew, D., Keel, M. K., \& Woolard, K. D. (2018). Cancer in wildlife: patterns of emergence. Nature Reviews Cancer, 18 (10), 646-661. doi:10.1038/s41568-018-0045-0

Peto, R., Roe, F. J., Lee, P. N., Levy, L., \& Clack, J. (1975). Cancer and ageing in mice and men. $B r J$ Cancer, 32 (4), 411-426. doi:10.1038/bjc.1975.242

Revell, L. J. (2012). phytools: an R package for phylogenetic comparative biology (and other things). Methods in Ecology and Evolution, 3 (2), 217-223. doi:10.1111/j.2041-210X.2011.00169.x

Risques, R. A., \& Promislow, D. E. L. (2018). All's well that ends well: why large species have short telomeres. Philos Trans R Soc Lond B Biol Sci, 373 (1741). doi:10.1098/rstb.2016.0448

Scherf, B. D. (2000). World Watch List for Domestic Animal Diversity (3 ed.). Rome, Italy: Food and Agriculture Organization of the United Nations.

Seluanov, A., Chen, Z., Hine, C., Sasahara, T. H. C., Ribeiro, A. A. C. M., Catania, K. C., . . . Gorbunova, V. (2007). Telomerase activity coevolves with body mass not lifespan. Aging Cell, 6 (1), 45-52. doi:10.1111/j.1474-9726.2006.00262.x

Seluanov, A., Gladyshev, V. N., Vijg, J., \& Gorbunova, V. (2018). Mechanisms of cancer resistance in long-lived mammals. Nature Reviews Cancer, 18 (7), 433-441. doi:10.1038/s41568-018-0004-9

Seluanov, A., Hine, C., Bozzella, M., Hall, A., Sasahara, T. H. C., Ribeiro, A. A. C. M., . . Gorbunova, V. (2008). Distinct tumor suppressor mechanisms evolve in rodent species that differ in size and lifespan. Aging Cell, 7 (6), 813-823. doi:10.1111/j.1474-9726.2008.00431.x

Shay, J. W., \& Wright, W. E. (2010). Telomeres and telomerase in normal and cancer stem cells. Febs Letters, 584 (17), 3819-3825. doi:10.1016/j.febslet.2010.05.026

Sridevi, V., Uma, K. D., Sivaramakrishnan, S., \& Isola, N. R. (2002). Telomere length as related to chromosome length in the genusPennisetum . Cytologia, 67 (2), 185-190.

Sudyka, J. (2019). Does reproduction shorten telomeres? Towards integrating individual quality with lifehistory strategies in telomere biology. Bioessays, 41 (11), e1900095. doi:10.1002/bies.201900095

Sudyka, J., Arct, A., Drobniak, S., Gustafsson, L., \& Cichoń, M. (2016). Longitudinal studies confirm faster telomere erosion in short-lived bird species. Journal of Ornithology, 157 (1), 373-375. doi:10.1007/s10336015-1304-4

Tacutu, R., Thornton, D., Johnson, E., Budovsky, A., Barardo, D., Craig, T., . . . de Magalhaes, J. P. (2018). Human Ageing Genomic Resources: new and updated databases. Nucleic Acids Research, 46 (D1), D1083-d1090. doi:10.1093/nar/gkx1042. Accessed 1 July 2020.

Tian, X., Doerig, K., Park, R., Can Ran Qin, A., Hwang, C., Neary, A., . . . Gorbunova, V. (2018). Evolution of telomere maintenance and tumour suppressor mechanisms across mammals. Philosophical Transactions of the Royal Society B: Biological Sciences, 373 (1741), 20160443. doi:10.1098/rstb.2016.0443

Tidière, M., Gaillard, J.-M., Berger, V., Müller, D. W. H., Bingaman Lackey, L., Gimenez, O., . . Lemaitre, J.-F. (2016). Comparative analyses of longevity and senescence reveal variable survival benefits of living in zoos across mammals. Scientific Reports, 6 , 36361-36361. doi:10.1038/srep36361 
Tricola Gianna, M., Simons Mirre, J. P., Atema, E., Boughton Raoul, K., Brown, J. L., Dearborn Donald, C., . . Haussmann Mark, F. (2018). The rate of telomere loss is related to maximum lifespan in birds.Philosophical Transactions of the Royal Society B: Biological Sciences, 373 (1741), 20160445. doi:10.1098/rstb.2016.0445

von Zglinicki, T. (2002). Oxidative stress shortens telomeres. Trends in Biochemical Sciences, 27 (7), 339-344. doi:10.1016/s0968-0004(02)02110-2

Weinstein, B. S., \& Ciszek, D. (2002). The reserve-capacity hypothesis: evolutionary origins and modern implications of the trade-off between tumor-suppression and tissue-repair. Experimental gerontology, 37 (5), 615-627. doi:https://doi.org/10.1016/S0531-5565(02)00012-8

Whittemore, K., Vera, E., Martínez-Nevado, E., Sanpera, C., \& Blasco, M. A. (2019). Telomere shortening rate predicts species life span.Proceedings of the National Academy of Sciences, 116 (30), 201902452. doi:10.1073/pnas.1902452116

Wilkins, A. S., Wrangham, R. W., \& Fitch, W. T. (2014). The "domestication syndrome" in mammals: a unified explanation based on neural crest cell behavior and genetics. Genetics, 197 (3), 795-808. doi:10.1534/genetics.114.165423

Wright, W. E., \& Shay, J. W. (2005). Telomere biology in aging and cancer. Journal of the American Geriatrics Society, 53 (9s), S292-S294. doi:10.1111/j.1532-5415.2005.53492.x

Data Accessibility: Species trait data is included in Tables S9 and S10 in the Supporting Information. The phylogenetic data is available within the R package 'PhyloOrchard' (O'Meara et al., 2012).

Author Contributions: M.L.P. analyzed the data and both authors designed research and wrote the paper.

\section{TABLES AND FIGURES}

Table 1: Phylogenetic multiple regressions with results from Gomes et al. (2011) compared to our reanalysis. ${ }^{+}$

\begin{tabular}{lll}
\hline & Gomes et al. & Our re-analysis \\
\hline Response: $\log _{10}($ mass $)$ & $(\mathrm{p}$-value $)$ & $\beta(\pi-\alpha \lambda \cup \boldsymbol{\varepsilon})$ \\
telomerase activity & $(0.0082)^{* *}$ & $-0.0014(0.890)$ \\
$\log _{10}($ telomere length $)$ & $(0.71)$ & $-1.8435(0.007)^{* *}$ \\
Response: $\log _{10}($ lifespan $)$ & & \\
telomerase activity & $(0.34)$ & $0.0039(0.029)^{*}$ \\
$\log _{10}($ telomere length $)$ & $(0.0032)^{* *}$ & $-0.4118(0.002)^{* *}$ \\
$\log _{10}($ mass $)$ & $(<0.0001)^{* * *}$ & $0.1401(<0.001)^{* * *}$ \\
$\mathrm{p}<0.05,{ }^{* *} \mathrm{p}<0.01,{ }^{* * *} \mathrm{p}<0.001$ & $* \mathrm{p}<0.05,{ }^{* *} \mathrm{p}<0.01,{ }^{* * *} \mathrm{p}<0.001$ & $* \mathrm{p}<0.05,{ }^{* *} \mathrm{p}<0.01,{ }^{* * *} \mathrm{p}<0.001$ \\
\hline
\end{tabular}

${ }^{+}$Gomes et al. do not explicitly describe their model parameters, but these are our best guess at a reconstruction. More complete results from our re-analyses are given in Table S1 in the Supporting information. 



Figure 1: Associations between telomere length, maximum lifespan and body mass (all $\log _{10}$-transformed) and telomerase activity (untransformed) across 57 mammal species. Bivariate phylogenetic regressions of these associations are shown. Scatter plots do not depict the phylogenetic controls. Domesticated species are shown in blue.

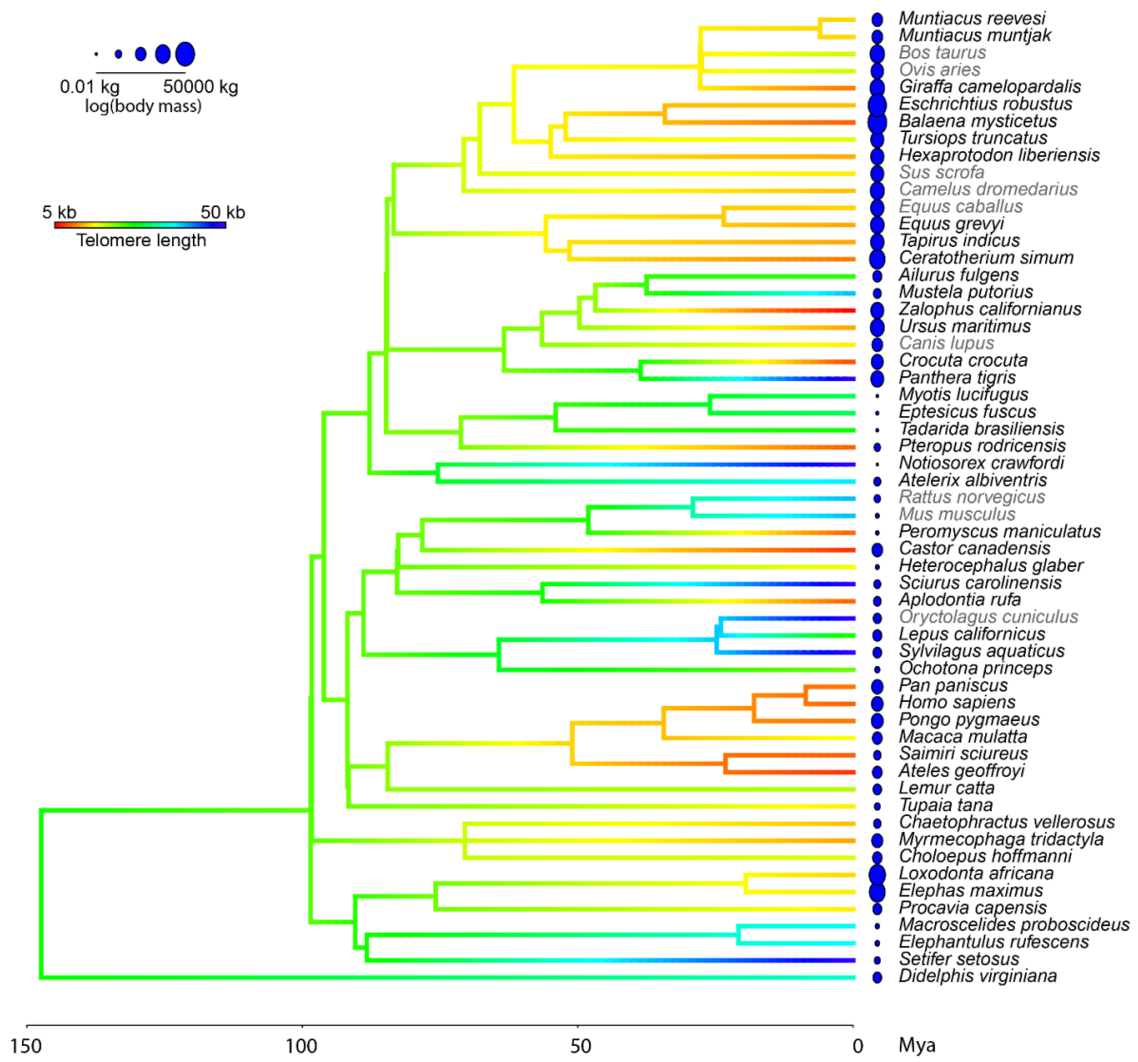

Figure 2: Phylogeny of 57 mammal species included in Gomes et al. (2011) illustrating the co-evolution of telomere length (in kb, red: shorter telomeres, blue: longer telomeres) and body mass ( $\log _{10}$-transformed, corresponding to the size of the blue spheres). Domesticated species are marked in gray. The timescale shows divergence estimates in million years ago (Mya). 

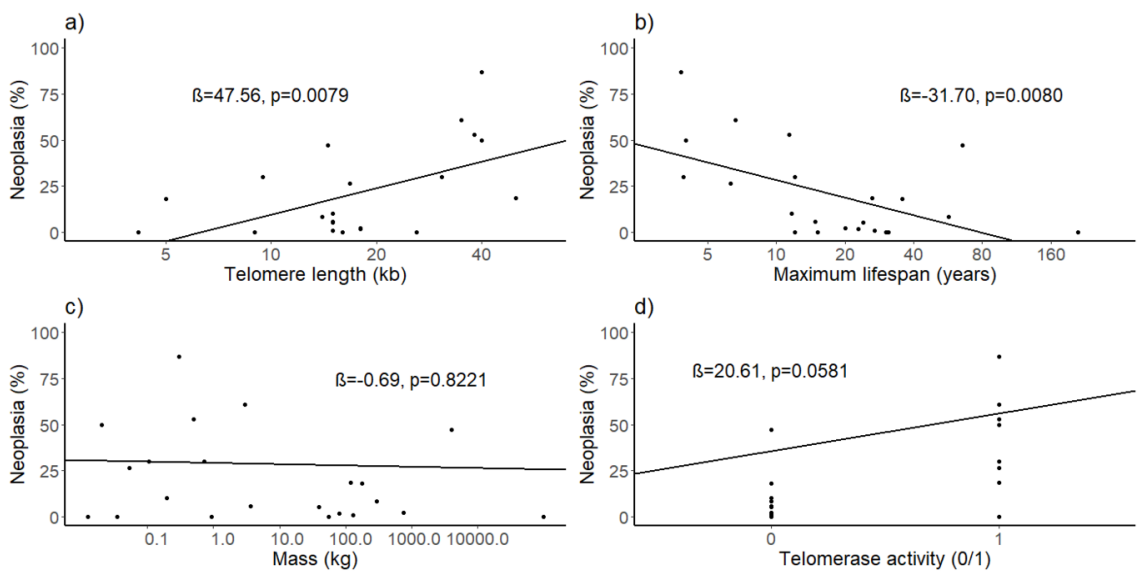

Figure 3: Associations between neoplasia incidence rates and a) telomere length, b) maximum lifespan, c) body mass (all $\log _{10}$-transformed) and d) telomerase activity (untransformed) across 22 mammal species. Bivariate phylogenetic regressions of these associations are shown. Scatter plots do not depict the phylogenetic controls.

Table 2: Phylogenetic multiple regression of neoplasia incidence rate predicted by telomere length and binary telomerase expression (0/1) across 22 mammal species.

\begin{tabular}{llr}
\hline Response: neoplasia rate & Estimate & Std. er \\
\hline intercept & -31.0668 & 20.464 \\
$\log _{10}$ (telomere length) & 36.2935 & 17.686 \\
binary telomerase activity (1) & 14.0411 & 9.8713 \\
$\lambda=0.000(95 \%$ CI: $0.000,0.963)$, adjusted $\mathrm{R}^{2}=0.3071$ & $\lambda=0.000(95 \%$ CI: $0.000,0.963)$, adjusted $\mathrm{R}^{2}=0.3071$ & $\lambda=0$. \\
$+\mathrm{p}<0.10$ & $+\mathrm{p}<0.10$ & $\mathrm{p}<$ \\
\hline
\end{tabular}

Table 3: Phylogenetic multiple regressions of log-transformed telomere length (TL) predicted by whether a species has been domesticated (1) or not (0), while accounting for telomerase activity (\%), body mass and lifespan across 57 mammal species.

\begin{tabular}{llr}
\hline Response: $\log _{10}(\mathrm{TL})$ & Estimate & Std. er \\
\hline intercept & 1.7168 & 0.1960 \\
domestication (1) & 0.1416 & 0.0615 \\
telomerase activity & 0.0060 & 0.0015 \\
$\log _{10}($ lifespan) & -0.3611 & 0.1203 \\
$\log _{10}$ (mass) & -0.0099 & 0.0298 \\
$\lambda=1.000(95 \%$ CI: $0.000,1.000)$, adjusted $\mathrm{R}^{2}=0.4121$ & $\lambda=1.000(95 \%$ CI: $0.000,1.000)$, adjusted $\mathrm{R}^{2}=0.4121$ & $\lambda=1$. \\
$\mathrm{p}<0.05, * * \mathrm{p}<0.01, * * * \mathrm{p}<0.001$ & $* \mathrm{p}<0.05, * * \mathrm{p}<0.01, * * * \mathrm{p}<0.001$ & $* \mathrm{p}<$ \\
\hline
\end{tabular}

